\title{
VOLUME IN TERMS OF CONCURRENT CROSS-SECTIONS
}

\author{
HERBERT BUSEMANN
}

1. Of the two expressions

$$
|M|=\frac{1}{2} \int_{0}^{2 \pi} r^{2}(\omega) d \omega=\frac{1}{2} \int_{0}^{2 \pi}\left(\int_{-r(\omega-\pi / 2)}^{r(\omega+\pi / 2)}|\rho| d \rho\right) d(\cdot)
$$

for the area $|M|$ of a plane domain $M$, given in polar coordinates $\rho$, $\omega$ by the inequalities $0 \leq \rho \leq r(\omega), 0 \leq \omega \leq 2 \pi$, the first has the well-known extension

$$
|M|=\frac{1}{n} \int_{\Omega_{n}} r^{n}(u) d \omega_{u}^{n}
$$

to $n$ dimensions. Here $\Omega_{n}$ is the surface of the unit sphere in the $n$-dimensional Euclidean space, $d \omega_{u}^{n}$ is its area element at the point $u$, and $M$ is given by $0 \leq \rho \leq r(u), u \in \Omega_{n}$.

In the second expression, $|\rho|$ may be interpreted as (1-dimensional) volume of the simplex with one vertex at the origin $z$ and the other at a variable point $p=(\rho, \omega \pm \pi / 2)$ in the cross-section of $M$ with the line normal to $\omega$. The purpose of the present note is the proof and the application of the following extension of this second expression to $n-1$ sets $M_{1}, \ldots, M_{n-1}$ in $E_{n}$ :

$$
=\frac{(n-1) !}{2} \int_{\Omega_{n}}\left(\int_{M_{1}(u)} \cdots \int_{M_{n-1}(u)} T\left(p_{1}, \cdots, p_{n-1}, z\right) d V_{p_{1}}^{n-1} \cdots d V_{p_{n-1}}^{n-1}\right) d \omega_{u}^{n}
$$

Here $M_{j}(u)$ is the cross-section of $M_{j}$ with the hyperplane $H(u)$ through $z$ normal to the unit vector $u$, the point $p_{j}$ varies in $M_{j}(u)$, the differential $d V_{p_{j}}^{n-1}$ is the $((n-1)$-dimensional $)$ volume element of $M_{j}(u)$ at $p_{j}$, and $T\left(p_{1}, \cdots, p_{n-1}, z\right)$ is the volume of the simplex with vertices $p_{1}, \cdots, p_{n-1}, z$.

Received November 19, 1951.

Pacific J. Math. 3 (1953), 1- 12 
Replacing the sets $M_{n-r+1}, \cdots, M_{n-1}^{4}$ by the unit sphere $U_{n}$ with center $z$ yields expressions for $\left|M_{1}\right| \cdots\left|M_{n-r}\right|$ in terms of the volume $T\left(p_{1}, \cdots, p_{n-r}, z\right)$, in particular ( 1 ) for $r=n-1$.

With the notation

$$
\kappa_{n}=\left|U_{n}\right|=\pi^{n / 2} / \Gamma\left(\frac{n}{2}+1\right),
$$

Steiner's symmetrization leads from (2) to the following result:

If $M_{1}, \ldots, M_{n-1}$ are convex bodies in $E_{n}(n \geq 3)$ with interior points, $z$ is a given point in $E_{n}$, and $M_{j}(u)$ the cross-section of $M_{j}$ with the plane normal to $u$ and through $z$, then

$$
\left|M_{1}\right| \ldots\left|M_{n-1}\right| \geq \frac{1}{n} \frac{\kappa_{n}^{n-2}}{\kappa_{n-1}^{n}} \int_{\Omega_{n}}\left|M_{1}(u)\right|^{n /(n-1)} \ldots\left|M_{n-1}(u)\right|^{n /(n-1)} d \omega_{u}^{n}
$$

and the equality sign holds only when the $M_{j}$ are homothetic ellipsoids with center $z$.

It follows in particular for a convex body $M$ that, for $n \geq 3$,

$$
|M|^{n-1} \geq \frac{1}{n} \frac{\kappa_{n}^{n-2}}{\kappa_{n-1}^{n}} \int_{\Omega_{n}}|M(u)|^{n} d \omega_{u}^{n}
$$

with the equality (if $|M|>0$ ) only for ellipsoids with center $z$. The efforts to prove this inequality, which has applications in Finsler spaces, led to the present investigation. The-because of Jensen's inequality-weaker estimate

$$
|M| \geq \frac{1}{n} \kappa_{n}^{-n /(n-1)} \int_{\Omega_{n}}|M(u)|^{n /(n-1)} d \omega_{u}^{n},
$$

with equality sign (if $|M|>0$ ) only for the spheres with center $z$, was found previously by L. A. Santaló who communicated it to the author. It is also the special case $M_{n-1}=M, M_{j}=U_{n}$ for $j<n-1$, of (3).

2. Let $M_{1}, \ldots, M_{n-1}$ be bounded Jordan measurable sets in $E_{n}, n>3$, such that the intersection of $l_{j}$ with any 1 -dimensional linear subspace (which in the future will be indicated by $L_{\nu}$ ) through a fixed given point $z$ possesses a 
$\nu$-dimensional Jordan measure. Since the subscripts run sometimes from 1 to $n$ and other times from 1 to $n-1$, we agree to use $\alpha, \beta$ for the former type, and $j, k$ for the latter, and may then omit mentioning the range.

I et $x_{x}$ be rectangular coordinates in $E_{n}$ with $z$ as origin. Take $n-1$ copies $E_{n}^{j}$ of $L_{n}$ with coordinates $x_{a}^{j}$, and let $M_{j}^{\prime}$ be the image of $M_{j}$ in $E_{n}^{j}$; that is, $x^{j} \in M_{j}^{\prime}$ if and only if the point $x$ with $x_{\alpha}=x_{\alpha}^{j}$ lies in $M_{j}$. Then $x_{a}^{j}$ may be considered as rectangular coordinates in the product space

$$
E=E_{n}^{\prime} \times \cdots \times E_{n}^{n-1}=\prod E_{n}^{j}
$$

hence

$$
\prod\left|M_{j}\right|=\prod\left|M_{j}^{\prime}\right|=\int_{\Pi M_{j}^{\prime}} d x_{1}^{1} \cdots d x_{n}^{1} \cdots d x_{1}^{n-1} \cdots d x_{n}^{n-1}
$$

In $E$ we introduce new coordinates

$$
\bar{x}_{1}^{1}, \ldots, \bar{x}_{n-1}^{1}, v_{1}, \ldots, \bar{x}_{1}^{n-1}, \ldots, \bar{x}_{n-1}^{n-1}, v_{n-1}
$$

through the relations

$$
x_{k}^{j}=\bar{x}_{k}^{j}, x_{n}^{j}=v_{1} \bar{x}_{1}^{j}+\cdots+v_{n-1} \bar{x}_{n-1}^{j}
$$

These equations fail to define $v_{j}$ if $\left|x_{k}^{j}\right|=\left|\bar{x}_{k}^{j}\right|=0$, i.e. if the points $x^{j}$ in $t_{n}$ are contained in an $L_{\nu}$ with $\nu<n-2$, or if the $L_{n-2}$ spanned by the $x^{j}$ is parallel to the $x_{n}$-axis. The geometric meaning of the right side of (2) shows that a special discussion of this case is superfluous.

To evaluate $\Pi\left|\left(M_{j}^{\prime}\right)\right|$ in the new coordinates, observe that the first $n$ rows in the $n(n-1)$-rowed Jacobian $J$ of the transformation (7) are in blocks of $n \times n$ matrices:

$$
\begin{array}{ccccccccccccccc}
1 & 0 & \ldots & 0 & 0 & 0 & 0 & \ldots & 0 & 0 & 0 & 0 & \ldots & 0 & 0 \\
0 & 1 & \ldots & 0 & 0 & 0 & 0 & \ldots & 0 & 0 & 0 & 0 & \ldots & 0 & 0 \\
. & \cdot & \ldots & . & . & \cdot & . & \ldots & \cdot & . & \cdot & \cdot & \ldots & 0 & 0 \\
0 & 0 & \ldots & 1 & 0 & 0 & 0 & \ldots & 0 & 0 & 0 & 0 & \ldots & 0 & 0 \\
v_{1} & v_{2} & \ldots & v_{n-1} & \bar{x}_{1}^{1}, & v_{1} & v_{2} & \ldots & v_{n-1} & \bar{x}_{2}^{1}, & v_{1} & v_{2} & \ldots & v_{n-1} & \bar{x}_{n-1}^{1}
\end{array}
$$

hence 


$$
J=\left|\bar{x}_{k}^{j}\right|
$$

The unit normal $u$ in $E_{n}$ to the plane

$$
x_{n}=x_{1} v_{1}+\cdots+x_{n-1} v_{n-1}
$$

is, with $w=\left(1+v_{1}^{2}+\cdots+v_{n-1}^{2}\right)^{1 / 2}$, either

$$
u_{j}=v_{j} w^{-1 / 2}, u_{n}=-w^{-1 / 2} \text { or } u_{j}=-v_{j} w^{-1 / 2}, u_{n}=w^{-1 / 2} \text {. }
$$

. Then $w^{-1}=|\cos \theta|$, where $\theta$ is the angle between $u$ and the $x_{n}$-axis, so that $d \omega_{u}^{n}=w d u_{1} \cdots d u_{n-1}$ is the area element of $\Omega_{n}$. Here we disregard again planes parallel to the $x_{n}$-axis. Now

$$
\left|\frac{\partial u_{j}}{\partial v_{k}}\right|=w^{-3(n-1)}\left|\begin{array}{ccccc}
w^{2}-v_{1}^{2} & -v_{1} v_{2} & \cdots & -v_{1} v_{n-1} \\
-v_{2} v_{1} & w^{2}-v_{2}^{2} & \cdots & -v_{2} v_{n-1} \\
\cdot & \cdot & \cdots & \cdot \\
-v_{n-1} v_{1} & -v_{n-1} v_{2} & \cdots & w^{2}-v_{n-1}^{2}
\end{array}\right|
$$

Since all principal minors of the determinant $\left|-v_{j} v_{k}\right|$ of order greater than 1 vanish, it follows ( compare $[4, \mathrm{pp} .125,126]$ ) that

$$
\left|\frac{\partial u_{j}}{\partial v_{k}}\right|=w^{-3(n-1)}\left(w^{2(n-1)}-w^{2(n-2) \sum} v_{j}^{2}\right)=w^{-n-1}
$$

and

$$
d \omega_{u}^{n}=w^{1-n-1} d v_{1} \cdots d v_{n-1}=\left|\cos ^{n} \theta\right| d v_{1} \cdots d v_{n-1}
$$

The volume element $d V_{x^{j}}^{n-1}$ of the hyperplane $x_{n}^{j}=\sum x_{k}^{j} v_{k}$ is

$$
d y_{x^{j}}^{n-1}=d x_{1}^{j} \cdots d x_{n-1}^{j}|\sec \theta|
$$

If we now interpret the points $x^{1}, \ldots, x^{n-1}$ as lying in the same $E_{n}$, then (8) shows that $|J| /(n-1)$ ! is the volume of the projection of the simplex with vertices $x^{1}, \ldots, x^{n-1}, z$ on the plane $x_{n}=0$. Since these points determine a hyperplane $H(u)$ with normal $u$ through $z$, 


$$
(n-1) ! T\left(x^{1}, \ldots, x^{n-1}, z\right)=|J \sec \theta|
$$

Replacing $x^{j}$ by $p_{j}$, we briefly summarize the results (9), (10), (11) as

$$
d V_{p_{1}}^{n} \cdots d V_{p_{n-1}}^{n}=(n-1) ! T\left(p_{1}, \cdots, p_{n-1}, z\right) d V_{p_{1}}^{n-1} \ldots d J_{p_{n-1}}^{n-1} d \omega_{u}^{n} \cdot
$$

After observing that in (2) by integrating over $\Omega_{n}$ every $M^{j}(u)$ is counted twice (once for $u$ and once for $-u$ ), we see that the relation (2) follows from (12).

For brevity we introduce, for sets $M_{1}, \cdots, M_{r}$ in $E_{s}$ with $r \leq s$, the notation

$$
\tau_{s}\left(M_{1}, \ldots, M_{r}, z\right)=\int_{M_{1}} \cdots \int_{M_{r}} T\left(p_{1}, \cdots, p_{r}, z\right) d V_{p_{1}}^{s} \ldots d V_{p_{s}}^{s} \text {, }
$$

and may then write (2) in the form

$$
\left|M_{1}\right| \cdots\left|M_{n-1}\right|=\frac{(n-1) !}{2} \int_{\Omega_{n}} \tau_{n-1}\left(M_{1}(u), \ldots, M_{n-1}(u), z\right) d \omega_{u}^{n} .
$$

3. In order to obtain expressions for $\left|\left\|_{1}|\cdots|\right\|_{r}\right|$ with $r<n-1$, we replace successively $n_{n-1}, \ldots, n_{n-r+1}$ by the unit sphere $U_{n}$. The contribution of the latter sets to the right side of (13) can then be integrated out by using the following fact:

Let an $L_{\mu}, 0<\mu<n-1$, through the center $z$ of the unit sphere $U_{n-1}$ in $E_{n-1}$, intersect $U_{n-1}$ in $U_{\mu}$. For any point $q$ in $U_{n-1}$, denote by $r$ the distance $q z$, and by $\phi$ the angle between the ray $q z$ and the $L_{\mu}$. Then

$$
\int_{U_{n-1}} r|\sin \phi| d V_{q}^{n-1}=\frac{\omega_{\nu-1}}{\omega_{\nu}} \cdot \kappa_{n}, \quad \nu=n-\mu,
$$

where $\omega_{\nu}=\nu \cdot \kappa_{\nu}=2 \pi^{\nu / 2} \Gamma^{-1}(\nu / 2)$ is the area of the surface $\Omega_{\nu}$ of $U_{\nu}$, in particular $\omega_{1}=2$.

To prove (14), let the $L_{\nu-1}$ normal to the $L_{\mu}$ through $q$ intersect $U_{\mu}$ in $p$, and $U_{n-1}$ in the sphere $S_{\nu-1}$. If $\rho=p z$ then $S_{\nu-1}$ has radius $\sigma=\left(1-\rho^{2}\right)^{1 / 2}$. Then $s=p q=r|\sin \phi|$; hence

$$
\int_{U_{n-1}} r|\sin \phi| d V_{q}^{n-1}=\int_{U_{\mu}}\left(\int_{v_{v}-1} s d V_{q}^{\nu-1}\right) d V_{p}^{\mu},
$$

If $d \omega \frac{\nu-1}{q}$ denotes the area element of the $\Omega_{\nu-1}$ with center $p$ in the $L_{\nu-1}$ at the 
point $\bar{q}$ of the ray $p q$, then

$$
\int_{\nu-1} s d V_{q-1}^{\nu-1}=\int_{\nu-1} \int_{0}^{\sigma} s s^{\nu-2} d s d \omega_{\bar{q}}^{\nu-1}=\frac{\sigma^{\nu}}{\nu} \omega_{\nu-1}=\left(1-\rho^{2}\right)^{\nu / 2} \frac{\omega_{\nu-1}}{\nu} .
$$

Therefore, with a similar notation,

$$
\begin{aligned}
& \int_{U_{n-1}} r|\sin \phi| d V_{q}^{n-1}=\frac{\omega_{\nu-1}}{\nu} \int_{\Omega_{\mu}} \int_{0}^{1}\left(1-\rho^{2}\right)^{\nu / 2} \rho^{\mu-1} d \rho d \omega_{p}^{\mu} \\
& =\frac{\omega_{\nu-1} \omega_{\mu}}{\nu} \frac{\Gamma(\mu / 2) \Gamma(\nu / 2+1)}{2 \Gamma(\mu / 2+\nu / 2+1)}=\frac{\omega_{\nu-1} \omega_{\mu}}{\nu} \frac{\Gamma(\mu / 2) \nu / 2 \Gamma(\nu / 2)}{2 \Gamma(n / 2+1)}=\frac{\omega_{\nu-1}}{\omega_{\nu}} \kappa_{n} .
\end{aligned}
$$

Returning to (13), we replace $M_{n-1}$ by $U_{n}$. Then $M_{n-1}(u)$ becomes the $(n-1)$ dimensional unit sphere $U_{n}(u)$ in the hyperplane with normal $u$. If $\phi$ is the angle between the ray $z p_{n-1}$ and the $L_{n-2}$ spanned by $p_{1}, \cdots, p_{n-2}, z$, then, with $r=z p_{n-1}$,

$$
T\left(p_{1}, \cdots, p_{n-1}, z\right)=(n-1)^{-1} r|\sin \phi| T\left(p_{1}, \ldots, p_{n-2}, z\right)
$$

Hence, carrying out the integration over $U_{n}(u)$, by (14) we obtain

$$
\begin{aligned}
\left|M_{1}\right| \cdots\left|M_{n-2}\right| & \cdot \kappa_{n} \\
& =\frac{1}{2}(n-2) ! \frac{\omega_{1}}{\omega_{2}} \kappa_{n} \int_{\Omega_{n}} \tau_{n-1}\left(M_{1}(u), \ldots, M_{n-2}(u), z\right) d \omega_{u}^{n}
\end{aligned}
$$

or

(15) $\left|M_{1}\right| \cdots\left|M_{n-2}\right|$

$$
=\frac{(n-2) !}{2 \pi} \int_{\Omega_{n}} \tau_{n-1}\left(M_{1}(u), \cdots, M_{n-2}(u), z\right) d \omega_{u}^{n}
$$

If now $M_{n-2}$ is replaced by $U_{n}$, then because of (14) the factor

$$
\frac{1}{n-2} \frac{\omega_{2}}{\omega_{3}} \kappa_{n}
$$


is introduced on the right. Continuing in this manner leads to the general relation

$$
\left|M_{1}\right| \ldots\left|M_{n-r}\right|=\frac{(n-r) !}{\omega_{r}} \int_{\Omega_{n}} \tau_{n-1}\left(M_{1}(u), \ldots, M_{n-r}(u), z\right) d \omega_{u}^{n}
$$

The integrand occurs in many $I I(u)$, and it would be more natural to replace the integration over $\Omega_{n}$ by an integration over all $L_{n-r}$. The results of integral geometry [5] lead to such a reduction for general $r$; however, we restrict our attention here to the two simplest cases, where no new formulas of the type (12) are required.

It is clear that the last formula in the sequence (16),

$$
\left|W_{1}\right|=\frac{1}{\omega_{n}} \int_{\varrho_{n}} \tau_{n-1}\left(M_{1}(u), z\right) d \omega_{u}^{n}
$$

must be essentially identical with (1). Indeed, if $M_{1}$ can be represented in the form $0 \leq \rho \leq r(u), u \in \Omega_{n}$, and we write the induced representation of $M_{1}(u)$ in the form

$$
0 \leq \rho \leq r(v), v \in \Omega_{n-2}(u)=H(u) \cap \Omega_{n},
$$

then, with $p z=\rho$, we have

$$
\begin{aligned}
\tau\left(M_{1}(u), z\right) & =\int_{M_{1}(u)} \rho d V_{p}^{n-1}=\int_{\Omega_{n-1}(u)} \int_{0}^{r(v)} \rho \rho^{n-2} d \omega_{v}^{n-1} \\
& =\frac{1}{n} \int_{\Omega_{n-1}(u)} r^{n}(v) d \omega_{v}^{n-1}
\end{aligned}
$$

and

$$
\left|M_{1}\right|=\frac{1}{n \cdot \omega_{n-2}} \int_{\Omega_{n}}\left(\int_{\Omega_{n-1}(u)} r^{n}(v) d \omega_{v}^{n-2}\right) d \omega_{u}^{n}
$$

Now according to the results on cinematic measure on the sphere (see [5], for $n=3$ already [3]), integrating over the $v$-normal to $u$ first, and then over $u$, leads to the same result as integrating over the $H(w)$ that contain $v$, that is, those for which $w$ is normal to $v$, and then over $v$. The first of the latter two integrations yields $\omega_{n-2} r^{n}(v)$, and (1) follows. 
As second example, we indicate briefly the reduction of (15). Denoting by $L_{n-2}^{p}$ the $L_{n-2}$ spanned by $p_{1}, \cdots, p_{n-2}, z$, and by $M_{i}^{p}$ the intersection of $M_{i}$ with $L_{n-2}^{p}$, we obtain from $(12)$ that if $L_{n-2}^{p}$ lies in $H(u)$ and has there the normal $v$, then

$$
\begin{aligned}
& \tau_{n-1}\left(M_{1}(u), \cdots, M_{n-2}(u), z\right) \\
& =\int_{M_{1}(u)} \cdots \int_{M_{n-2}(u)} T\left(p_{1}, \cdots, p_{n-2}, z\right) d V_{p_{1}}^{n-1} \cdots d V_{p_{n-2}}^{n-1} \\
& =\frac{(n-2) !}{2} \int_{\Omega_{n-1}(u)} \int_{M_{1}^{p}} \cdots \int_{M_{n-2}^{p}} T^{2}\left(p_{1}, \cdots, p_{n-2}, z\right) d V_{p_{1}}^{n-2} \cdots d V_{p_{n-2}}^{n-2} d \omega_{v}^{n-1} .
\end{aligned}
$$

Substituting this in (15) leads besides the integrations over the $M_{i}^{p}$ to integrations over $\Omega_{n-1}(u)$ and $\Omega_{n}$. Similarly as in the preceding case, these latter two may be reduced to one integration by using the cinematic measure $d L_{n-2}^{p}$ on $\Omega_{n}$ of the $\Omega_{n-2}$ in which $L_{n-2}^{p}$ intersects $\Omega_{n}$ (compare [5]). The result (given without verification because it will not be used) is

$$
\left|M_{1}\right| \cdots\left|M_{n-2}\right|
$$

$$
=\frac{[(n-2) !]^{2}}{2} \int_{\Omega_{n}} \int_{M_{1}^{p}} \cdots \int_{M_{n-2}^{p}} T^{2}\left(p_{1}, \cdots, p_{n-2}, z\right) d V_{p_{1}}^{v-2} \cdots d V_{p_{n-2}}^{n-2} d L_{n-2}^{p} \cdot
$$

4. To obtain the estimate (3) we use Steiner's symmetrization in the form suggested by Blaschke's treatment of Sylvester's l'roblem (compare [1, 24]). In the following the subscripts $i, h$ run from 1 to $m$.

Let $M_{1}, \ldots, M_{m}$ be convex bodies with interior points in $E_{m}$. In an arbitrary system of rectangular coordinates with origin $z$, symmetrize each $M_{i}$ with respect to the $\left(x_{1}, \cdots, x_{m-1}\right)$-plane $P$; that is, slide a segment in which a line $L_{1}$ parallel to the $x_{m}$-axis intersects $M_{i}$ along $L_{1}$ such that its center falls on $P$. Call $\bar{M}_{i}$ the image of $M_{i}$ under this transformation, and $\bar{p}_{i}$ the image in $\bar{M}_{i}$ of a given point $p_{i}$ in $M_{i}$. The mapping preserves volume, $d V_{\bar{p}_{i}}^{m}=d V_{p_{i}}^{m}$. We are going to show that $\tau_{m}\left(M_{1}, \cdots, M_{m}, z\right)$ does not increase.

If $p_{i} \in M_{i}$, denote by $p_{i}^{\prime}$ the point symmetric to $p_{i}$ with respect to the center of that chord of $M_{i}$ parallel to the $x_{m}$-axis which goes through $p_{i}$. If $p_{i}^{1}, \cdots, p_{i}^{m}$ are the coordinates of $p_{i}$, then with $\eta=1 / m$ !

$$
\pm T\left(p_{1}, \cdots, p_{m}, z\right)=\eta\left|p_{i}^{h}\right|, \pm T\left(p_{1}^{\prime}, \cdots, p_{m}^{\prime}, z\right)=\eta\left|p_{i}^{\prime h}\right|
$$


The images $\bar{p}_{i}$ of $p_{i}$ and $\bar{p}_{i}^{\prime}$ of $p_{i}^{\prime}$ satisfy the relation

$$
\left|\bar{p}_{i}^{h}\right|=-\left|\bar{p}_{i}^{\prime h}\right|
$$

hence

$$
2 T\left(\bar{p}_{1}, \ldots, \bar{p}_{m}, z\right)=2 T\left(\bar{p}_{1}^{\prime}, \ldots, \bar{p}_{m}^{\prime}, z\right)=\eta|| \bar{p}_{i}^{h}|-| \bar{p}_{i}^{\prime h}||
$$

But

$$
p_{i}^{h}=p_{i}^{\prime h}=\bar{p}_{i}^{h}=\bar{p}_{i}^{\prime h} \text { for } 1 \leq h \leq m-1 \text {, and } p_{i}^{m}-p_{i}^{\prime m}=\bar{p}_{i}^{m}-\bar{p}_{i}^{\prime m} \text {. }
$$

so that

$$
\left|\bar{p}_{i}^{h}\right|-\left|\bar{p}_{i}^{\prime h}\right|=\left|p_{i}^{h}\right|-\left|p_{i}^{\prime h}\right|
$$

hence

$$
T\left(p_{1}, \ldots, p_{m}, z\right)+T\left(p_{1}^{\prime}, \ldots, p_{m}^{\prime}, z\right) \geq 2 T\left(\bar{p}_{1}, \ldots, \bar{p}_{m}, z\right)
$$

Since

$$
\begin{aligned}
\tau_{m}\left(M_{1}, \ldots, M_{m}, z\right) & =\int_{M_{1}} \cdots \int_{M_{m}} T\left(p_{1}, \ldots, p_{m}, z\right) d V_{p_{1}}^{m} \cdots d V_{p_{m}}^{m} \\
& =\int_{M_{1}} \cdots \int_{M_{m}} T\left(p_{1}^{\prime}, \cdots, p_{m}^{\prime}, z\right) d V_{p_{1}^{\prime}}^{m} \cdots d V_{p_{m}^{\prime}}^{m},
\end{aligned}
$$

we conclude from (18) and

$$
d V_{\bar{p}_{i}}^{m}=d V_{p_{i}}^{m}, d V_{\bar{p}_{i}^{\prime}}^{m}=d V_{p_{i}^{\prime}}^{m}
$$

that

$$
\tau_{m}\left(M_{1}, \ldots, M_{m}, z\right) \geq \tau_{m}\left(\bar{M}_{1}, \ldots, \bar{M}_{m}, z\right)
$$

To discuss the equality sign, consider points $p_{i}$ in $M_{i}$ which are centers of chords parallel to the $x_{n}$-axis. Then $p_{i}=p_{i}^{\prime}$, and the points $\bar{p}_{i}=\bar{p}_{i}^{\prime}$ lie in $P$, so that the right side of (18) vanishes. Therefore the equality sign can hold in (18) only when the points $p_{1}, \cdots, p_{m}, z$ are coplanar. Choosing $p_{1}, \cdots, p_{i-1}$, 
$p_{i+1}, \cdots, p_{m}$ such that they and $z$ do not lie in an $L_{m-2}$ (the $M_{i}$ have interior points!) we see that all centers of chords of $M_{i}$ parallel to the $x_{m}$-axis must lie in the $L_{m-1}$ spanned by $p_{1}, \cdots, p_{i-1}, p_{i+1}, \cdots, p_{m}, z$. Moreover, this same $L_{m-1}$ must contain the centers of the chords parallel to the $x_{m}$-axis of all the other $M_{n}$. Thus we have proved:

(20) If $M_{1}, \cdots, M_{m}$ are convex bodies in $E_{m}$ with interior points, then simultaneous symmetrization of the $M_{i}$ with respect to any plane $P$ through $z$ decreases $\tau_{m}\left(M_{1}, \ldots, M_{m}, z\right)$ unless $z$ and the centers of the chords perpendicular to $P$ of all $M_{i}$ are coplanar.

For given positive values $\left|M_{1}\right|, \cdots,\left|M_{m}\right|$, the expression $\tau_{m}\left(\|_{1}, \ldots, M_{m}, z\right)$ can therefore be minimal only if the centers of every family of parallel chords of the different $M_{j}$ lie on the same plane through $z$. This implies, first, that each $M_{j}$ is an ellipsoid with center $z,{ }^{1}$ and then that all these ellipsoids are homothetic.

That the minimum is actually reached in this case is proved by the following standard argument ( see $[1, \S 24])$. Using a suitable sequence $P_{\nu}$ of planes through $z$, and symmetrizing $M_{1}, \ldots, M_{m}$ successively in $P_{1}, P_{2}, \ldots$, yields a sequence $M_{1}^{\nu}, \ldots, M_{m}^{\nu}$ of convex bodies which tend to spheres $S_{1}, \ldots, S_{m}$ with center $z$ and, of course, $\left|S_{i}\right|=\left|M_{i}^{\nu}\right|=\left|M_{i}\right|$ (compare [2, \&41]).

The functional $\tau_{m}\left(M_{1}, \ldots, M_{m}, z\right)$ is monotone [that is, $M_{i}^{\prime} \subset M_{i}$ implies $\left.\tau_{m}\left(M_{1}^{\prime}, \ldots, M_{m}^{\prime}, z\right) \leq \tau_{m}\left(M_{1}, \ldots, M_{m}, z\right)\right]$ and positive homogeneous:

$$
\tau_{m}\left(\lambda M_{1}, \ldots, \lambda M_{m}, z\right)=\lambda^{m(m+1)} \tau_{m}\left(M_{1}, \ldots, M_{m}, z\right) \text { for } \lambda>0 .
$$

For a given $\epsilon>0$, choose $N(\epsilon)>0$ such that $S_{i} \subset(1+\epsilon) M_{i}^{\nu}$ for $\nu>N(\epsilon)$ and all $i$. Then for $\nu>N(\epsilon)$, because of $(20)$ and the two mentioned properties, we have

$$
\begin{aligned}
\tau_{m}\left(S_{1}, \ldots, S_{m}, z\right) & \leq(1+\epsilon)^{m(m+1)} \tau_{m}\left(M_{1}^{\nu}, \ldots, M_{m}^{\nu}, z\right) \\
& \leq(1+\epsilon)^{m(m+1)} \tau_{m}\left(M_{1}, \ldots, M_{m}, z\right),
\end{aligned}
$$

which proves $\tau_{m}\left(S_{1}, \ldots, S_{m}, z\right) \leq \tau_{m}\left(M_{1}, \ldots, M_{m}, z\right)$ and hence the mini-

${ }^{1}$ The proofs found in the literature all refer to the cases $m=2,3$; for references $[2,\{70]$. However, the extension to arbitrary $m$ is immediate. A particularly simple proof, which works for all $m$ and is not found in the literature, is obtained by using Loewner's result, that there is exactly one ellipsoid which has a given center, contains a given convex body, and has minimal volume. 
mum property for homothetic ellipsoids with center $z$.

To evaluate $\tau_{m}\left(S_{1}, \cdots, S_{m}, z\right)$, denote the radius of $S_{i}$ by $r_{i}$. Then the results of section 3 show that

$$
\begin{aligned}
\tau_{m}\left(S_{1}, \ldots, S_{m}, z\right) & =\frac{1}{m} r_{m}^{m+1} \frac{\omega_{1}}{\omega_{2}} \kappa_{m+1} \tau_{m}\left(S_{1}, \ldots, S_{m-1}, z\right) \\
& =\frac{1}{m(m-1)} \frac{\omega_{1}}{\omega_{3}} r_{m}^{m+1} r_{m+1}^{m+1} \kappa_{m+1}^{2} \tau_{m}\left(S_{1}, \ldots, S_{m-2}, z\right)=\cdots \\
& =\frac{1}{m !} \prod r_{i}^{m+1} \frac{\omega_{1}}{\omega_{m+1}} \kappa_{m+1}^{m}=\frac{1}{m !} \frac{2}{\omega_{m+1}} \frac{\kappa_{m+1}^{m}}{\kappa_{m}^{m+1}} \prod\left|S_{i}\right|^{(m+1) / m} .
\end{aligned}
$$

Therefore we have:

(21) If $M_{1}, \ldots, M_{m}$ are convex bodies in $E_{m}$ with interior points, then

$$
\tau_{m}\left(M_{1}, \ldots, M_{m}, z\right) \geq \frac{2}{(m+1) !} \frac{\kappa_{m+1}^{m-1}}{\kappa_{m}^{m+1}} \prod\left|M_{i}\right|^{(m+1) / m},
$$

and the equality sign holds only for homothetic ellipsoids with center $z$.

Applying this result to (13) yields the inequalities (3) and (4) with the conditions for the equality sign. The latter result may also be formulated as follows:

Among all convex bodies $M$ with a given volume, the ellipsoids with center $z$ (and only these) maximize $\int_{\Omega_{n}}|M(u)|^{n} d \omega_{u}^{n}$.

To ask for the minimum is senseless since for any convex body $M$ the integral $\int_{\Omega_{n}}|M(u)|^{n} d \omega_{u}^{n}$ will tend to zero when $M$ moves to infinity. However, it is a meaningful, but unsolved, problem to find the minimum of this integral for all convex bodies with a given volume and center $z$. This is equivalent to the problem of finding the smallest constant $K$ such that for any convex $M$ with center $z$ the inequality

$$
K \int_{\Omega_{n}}|M(u)|^{n} d \omega_{u}^{n} \geq|M|^{n-1}
$$

holds. The existence of $K$ follows readily from (2). 
Finally (5) shows:

Among all convex bodies with center $z$ the spheres (and only these) yield the maximum of

$$
\min _{u}|M(u)|^{n}|M|^{1-n}
$$

The corresponding minimum maximum problem seems quite difficult.

\section{REFERENCES}

1. W. Blaschke, Vorlesungen über Differentialgeometrie, vol. II, Berlin, 1923, and New York, 1949.

2. T. Bonnesen und W. Fenchel, Theorie der konvexen Körper, Berlin, 1934, and New York, 1948.

3. P. Funk, Über Flächen mit lauter geschlossenen geodätischen Linien, Math. Ann. 74 (1913), 278- 300.

4. G. Kowalewski, Einführung in die Determinantenthe orie, Leipzig, 1909.

5. B. Petkantschin, Integralgeometrie 6. Zusammenhänge zwischen den Dichten der linearen Unterräume im n-dimensionalen Raum, Abh. Math. Sem. Univ. Hamburg 11 (1936), $249-310$.

University of Southern California 